\title{
¿El personal de salud responsable de la vigilancia epidemiológica sabe identificar a las enfermedades de notificación obligatoria?
}

\author{
Yamily Yrigoín-Pérez, ${ }^{1}$ Cristian Díaz-Vélez² y Moisés Apolaya-Segura ${ }^{3}$ \\ ${ }^{1}$ Sociedad Científica de Estudiantes de Medicina Veritas SCIEMVE; ${ }^{2}$ Oficina de Inteligencia Sanitaria, Hospital Nacional Almanzor Aguinaga Asenjo \\ EsSalud; '3niversidad de San Martín de Porres, Centro de Investigación en Epidemiología Clínica y Medicina Basada en Evidencias. Chiclayo, \\ Perú
}

Estimado editor,

En respuesta al artículo de Lugo-Caballero et al. ${ }^{1}$ sobre conocimiento de enfermedades transmitidas por vectores (dengue, rickettsiosis y enfermedad de Chagas) en médicos, donde se describe que $62.5 \%$ de los médicos presentó conocimiento moderado, $37.5 \%$ conocimiento pobre y $0 \%$ conocimiento adecuado me permito hacer algunos comentarios respecto al tema.

Lavigilancia epidemiológica de enfermedades infecciosasy daños sujetos a notificación obligatoria es crucial para la planificación, implementación y evaluación de programas de prevención y control de enfermedades, así como para identificar regiones y grupos poblacionales más comprometidos. ${ }^{2,3}$ Depende de la definición del caso y el reconocimiento de la enfermedad, la compilación de datos individuales, la notificación y análisis para la detección oportuna de brotes con la finalidad de controlar el impacto sobre la población. ${ }^{3}$ El proceso de vigilancia epidemiológica requiere personal de salud capacitado para presentar un informe detallado o abreviado de los casos de enfermedades de notificación obligatoria a las autoridades pertinentes. ${ }^{2}$

Estudios realizados en diferentes partes del mundo han demostrado que una de cada dos personas se sentía informada sobre la obligación de notificar, sin embargo, lo hacía de forma incompleta. Entre las causas más frecuentes para no cumplir con los requisitos de información están la falta de conocimientos para informar —no estar seguros de que la enfermedad diagnosticada es reportable-, la actitud negativa hacia los informes y la falta de motivación debido a la inadecuada retroalimentación. ${ }^{3-5}$

Actualmente, en Perú existen 19 enfermedades infecciosas de vigilancia epidemiológica, de las cuales 12 se han reportado en la región Lambayeque. ${ }^{6}$ La mayoría del personal de salud obtiene sus conocimientos de programas de televisión, internet y experiencias de otros colegas; sin embargo, se ha reportado que $50 \%$ tiene conocimiento deficiente sobre vigilancia epidemiológica. ${ }^{3}$

El Seguro Social de Perú tiene una cobertura aproximada de $34 \%^{7}$ de la población nacional y durante septiembre de 2018 se realizó la reunión nacional anual con los responsables de la vigilancia epidemiológica de las 33 redes que lo integran, a quienes se le aplicó la sección de "Conocimientos" del Cuestionario de Conocimientos sobre Vigilancia Epidemiológica, Actitudes en la Notificación Epidemiológica y Experiencia en Reporte de Enfermedades de Notificación (validado por expertos y con KR-20 de 0.71 para conocimientos y alfa de Cronbach de 0.76 para actitudes). ${ }^{8}$ Dicho cuestionario plantea 12 preguntas, escenarios y casos-problema para identificar enfermedades de notificación obligatoria. Fue autoadministrado y respondido por 36 profesionales de salud (Tabla 1).

En los resultados se observó que la mayoría del personal responsable de vigilancia epidemiológica había tenido capacitación, sin embargo, solamente $5.5 \%$ respondió correctamente al cuestionario y más de $50 \%$ tuvo ocho o menos aciertos.

Desde 2013 se han incrementado los casos notificados de dengue. En 2014 se alertó sobre la precaución de sarampión y rubéola, debido al incremento del flujo poblacional entre países: en 2015 se notificó un caso de sarampión importado de Alemania, casos importados de chikunguña y el primer caso autóctono de esta última; también se reportaron casos de rabia
Correspondencia:

Cristian Díaz-Vélez

E-mail: cristiandiazv@ hotmail.com
Fecha de recepción: 12-02-2018

Fecha de aceptación: 13-02-2018

DOI://dx.doi.org/10.24875/GMM.18004216
Gac Med Mex. 2018;154:352-353

Disponible en PubMed

www.gacetamedicademexico.com 
Tabla 1. Conocimientos sobre proceso de vigilancia epidemiológica y notificación obligatoria de enfermedades infecciosas en personal de salud $(n=36)$

\begin{tabular}{lcc}
\hline Característica & $\mathbf{n}$ & $\%$ \\
\hline Capacitación en vigilancia epidemiológica & & \\
Sí & 25 & 69.4 \\
No & 11 & 30.6 \\
Frecuencia de respuestas por cada pregunta & & \\
del cuestionario aplicado & & \\
Todas las preguntas & 2 & 5.5 \\
10 acierto & 3 & 8.3 \\
9 aciertos & 12 & 33.3 \\
8 aciertos & 10 & 27.8 \\
7 aciertos & 4 & 11.1 \\
6 acierto & 3 & 8.3 \\
5 aciertos & 2 & 5.6 \\
& & \\
Reconocimiento de casos clínicos & & \\
Enfermedad de notificación obligatoria & 31 & 86.1 \\
Dengue & 29 & 85.6 \\
Cólera & 35 & 97.2 \\
Malaria & 5 & 13.9 \\
Rubéola & 12 & 33.3 \\
Peste neumónica & 29 & 80.6 \\
Poliomielitis & 35 & 97.2 \\
Acciones ante caso notificable & & \\
Realiza la notificación obligatoria & \\
Reconoce quién es el responsable de & 12 & 13.9 \\
realizar la notificación obligatoria en su & 26 & 72.3 \\
establecimiento de salud & 36 & 100.0 \\
Identifica reporte de notificación Internacional & & \\
Reconoce nexo epidemiológico & & \\
\hline
\end{tabular}

humana y diseminación de rabia canina en el departamento de Puno y Arequipa. Ese mismo año se avisó del incremento de riesgo de transmisión de influenza y otros virus respiratorios en aproximadamente $12.8 \%$ (http://www.dge.gob.pe/salasituacional). Durante 2017, en el Perú se han identificado diversos brotes de dengue, malaria, zika, chikunguña, peste bubónica y otras enfermedades de vigilancia epidemiológica. ${ }^{6,8}$

Según la encuesta referida hubo mayor déficit de reconocimiento a malaria (84\%), rubéola (67\%), dengue (19\%) y peste neumónica (19\%); al comparar los resultados con las estadísticas podemos afirmar que existe un número subestimado de casos de enfermedades infecciosas de notificación obligatoria y, por lo tanto, de brotes no identificados. ${ }^{6,8}$

Asimismo, se evidenció que solo $13.9 \%$ de los encuestados realizaba la notificación obligatoria y $33.3 \%$ reconocía a los responsables de realizar la notificación obligatoria en su establecimiento de salud, hecho que puede relacionarse con las causas encontradas por Nader et al., ${ }^{2}$ Tan et al. ${ }^{5}$ y Mera et al. ${ }^{3}$

El retraso en la identificación de las enfermedades infecciosas de vigilancia epidemiológica conlleva a que en Perú la intervención del Ministerio de Salud no sea la más óptima y aparezcan brotes y epidemias que pueden ocasionar la muerte de numerosas personas; por ello, es importante que el personal encargado de los puestos de salud esté en constante capacitación y actualización para el reconocimiento oportuno de las diferentes enfermedades infecciosas de vigilancia epidemiológica y conozca la forma correcta de realizar su notificación obligatoria.

\section{Bibliografía}

1. Lugo-Caballero CI, Dzul-Rosado K, Dzul-Tut I, Balam-May Á, Zav-la-Castro J. Conocimiento de enfermedades transmitidas por vectores (dengue, rickettsiosis y enfermedad de Chagas) en médicos. Gac Med Mex. 2017;153:321-328.

2. Nader F, Askarian M. How do Iranian physicians report notifiable diseases? The first report from Iran. Am J Infect Control. 2009;37(6):500-504.

3. Mera-Muguerza C, Díaz-Vélez C. Nivel de conocimientos y actitudes sobre proceso de vigilancia epidemiológica y notificación obligatoria de enfermedades infecciosas en personal de salud en primer nivel en la provincia de Chiclayo [Tesis para obtener el título profesional de médico cirujano]. Perú: Universidad de San Martín de Porres; 2017.

4. Nnebue CC, Onwasigwe CN, Adogu POU, Onyeonoro UU. Awareness and knowledge of disease surveillance and notification by health-care workers and availability of facility records in Anambra state, Nigeria. Niger Med J J Niger Med Assoc. octubre de 2012;53(4):220-5.

5. Hsiu-Fen T, Chia-Yu Y, Hsueh-Wei C, Chen-Kang C, Hung-Fu Tseng. Private doctors' practices, knowledge, and attitude to reporting of communicable diseases: a national survey in Taiwan. BMC Infect Dis. 2009;9:11.

6. Ministerio de Salud. Boletín Epidemiológico del Perú. Perú: Ministerio de Salud; 2017.

7. EsSalud. Características demográficas, sociales, económicas y acceso a los servicios de salud de la población asegurada a EsSalud, 2015. Perú: Seguro Social de Salud; 2015.

8. Centro Nacional de Epidemiología, Prevención y Control de Enfermedades. [Sitio web]. Alertas epidemiológicas 2017. [Actualizado 2018]. Perú: Ministerio de Salud; Disponible en: http://www.cdc.gob.pe/portal/index. php?option=com_content\&view=article\&id=448. 